\title{
Sex differences in patients with high risk HPV-associated and HPV negative oropharyngeal and oral cavity squamous cell carcinomas
}

\author{
Hong Li ${ }^{1}$, Henry S. Park ${ }^{1,2,4}$, Heather A. Osborn ${ }^{1,3,4}$ and Benjamin L. Judson ${ }^{1,3,4^{*}}$
}

\begin{abstract}
Background: Human papilloma virus (HPV)-associated head and neck cancer is now recognized as a distinct clinical entity from HPV-negative tumors, which are primarily associated with tobacco and alcohol exposure.Little is known, however, about the behavior of HPV-associated oropharynx (OP) and oral cavity (OC) SCCs as two distinct cancers and how sex affects the overall survival (OS) in these two cancers. The objective of our study is to determine if sex is associated with overall survival (OS) in patients with high-risk human papillomavirus (HPV)-positive and HPV-negative squamous cell carcinomas (SCC) in the oropharynx and oral cavity sites.

Methods: This is a retrospective cohort study using a national database. Data were extracted from the National Cancer Database (NCDB) of patients diagnosed with OP or OC SCC from 2010 to 2014. Univariate and multivariate survival analyses were conducted with chi-square tests, Kaplan-Meier estimates, log-rank tests, and Cox proportional hazards multivariable modeling.

Results: A total of 30,707 patients (13,694 OP HPV-associated, 7933 OP HPV-, 1220 OC HPV-associated, 7860 OC HPV-) were identified. In all four groups, women tended to be older and have lower T and N clinical classification than men. Though there were no significant differences in OS between the sexes in OP HPV-associated cancers, female sex was associated with worse OS in OP HPV- cancers (HR: 1.15; 95\% Cl 1.04-1.28, $p=0.004$ ), whereas it was associated with improved OS in OC HPV-associated and HPV- cancers (HPV-associated: HR: 0.71; 95\% Cl 0.50-0.99, $p=0.048$; HPV-: HR: 0. $87 ; 95 \% \mathrm{Cl} 0.78-0.95, p=0.004)$.

Conclusion: The effect of sex on OS in OC and OP SCC appears to vary based on tumor location and HPV status. While the source of this difference in prognostic association is unclear, it may be related to an emerging difference in the biology of HPV carcinogenesis in these locations.
\end{abstract}

Keywords: Human papilloma virus, Sex difference, Head and neck cancer, Oropharyngeal cancer, Oral cavity cancer

\section{Background}

In the last 15 years, evidence has amassed on the human papilloma virus (HPV) as an important cause of head and neck squamous cell carcinomas (HNSCC). HPV-associated HNSCC is now recognized as a distinct clinical entity from HPV-negative HNSCC tumors [1], which are primarily associated with tobacco and alcohol exposure [2].

\footnotetext{
* Correspondence: benjamin.judson@yale.edu

${ }^{1}$ Yale School of Medicine, New Haven, CT, USA

${ }^{3}$ Department of Surgery (Section of Otolaryngology), Yale New Haven

Hospital, New Haven, CT, USA

Full list of author information is available at the end of the article
}

Given this recent discovery, many questions still remain regarding the epidemiology and management of patients with HPV-associated HNSCC. A subset of HNSCCs occurs in the oropharynx (OP). Chaturvedi and colleagues found that the incidence of OP cancers have been rising 1-2\% every year from 1973 to 2004 [3]. Despite HPV infection being common in both men and women, the incidence of HPV-associated OPSCCs is more than two-fold higher among men than women [4]. This sex-specific finding raises questions regarding possible differences in the biological presentation of the cancer between men and women. 
OPSCCs are now hypothesized to behave distinctly compared to HNSCCs at other sites. HPV DNA has been discovered in tumors from other head and neck sites such as cancers of the oral cavity (OC) [5-7]. A recent study found that HPV-associated non-OPSCCs display a distinct immune microenvironment and clinical behavior compared to HPV-associated OPSCCs [8].

To date, few studies have alluded to the sex-related differences in the prognosis for OPSCCs and other HNSCCs. One retrospective, multi-institutional study [9] found sex to be a significant prognostic factor for overall survival (OS) in OPSCCs even after accounting for HPV status. Interestingly, the same study found that in non-OPSCCs, sex did not have any prognostic significance for OS.

Many studies in HPV-associated HNSCCs have examined all HNSCCs as a whole entity $[10,11]$. Little is known, however, about the behavior of HPV associated OP and OC SCCs as two distinct cancers and how sex affects the OS in these two cancers. Therefore, this study aims to classify patient characteristics and investigate survival differences by sex in patients with HPV-associated and HPV- OPSCCs and OCSCCs.

\section{Methods}

Data

Data were extracted from the National Cancer Database (NCDB) from 2010 to 2014. The NCDB is a joint project of the Commission on Cancer and the American Cancer Society [12]. Cases are recorded from over 1500 accredited hospitals in the United States and Puerto Rico. The database represents over $70 \%$ of incident cases of cancer in the United States. Each hospital that participates in the registry is responsible for submitting and tracking patient and tumor level data on patients with malignant neoplastic diseases.

\section{Patient population}

Our study population includes patients whose primary malignancy was diagnosed as squamous cell carcinoma of the oropharynx or oral cavity. The following Internal Classification of Disease for Oncology, Third Edition (ICD-O-3) histology codes were used for squamous cell carcinoma M8070-8073 and the following topography codes were used for oropharynx (OP): C09.0-09.1, C09.8-09.9 (tonsil) C10.0, C10.2-10.4 (other oropharynx) and C-01.9 (base of tongue), for oral cavity (OC) cancer C00.0-00.9 (lip), C02.0-02.4, C02.8-02.9 (other/unspecified parts of the tongue), C03.0-03.1, C03.9 (gum), C04.0-04.1, C04.8-04.9 (floor of mouth), C05.0-05.1, C05.8-05.9 (palate), C06.006.2, C06.8-06.9 (other/unspecified parts of the mouth).

HPV status was available for cases diagnosed 2010-2014 and was categorized as negative, positive for low-risk HPV types, positive for high-risk HPV types (HPV 16 and/or 18) and HPV status unknown. For our study, patients were classified as 'HPV-positive' if they tested positive for high-risk HPV types, and 'HPV-negative' if they received a negative HPV test. Patients with low-risk HPV types or unknown HPV status were excluded.

We examined patient demographic and tumor data (age at diagnosis, race, Charlson/Deyo score, primary tumor site, American Joint Commission on Cancer $\mathrm{T}$ and $\mathrm{N}$ classification, lymph node metastasis, primary treatment type, insurance status, median income quartiles, treatment facility type and location, and rural/urban classification of

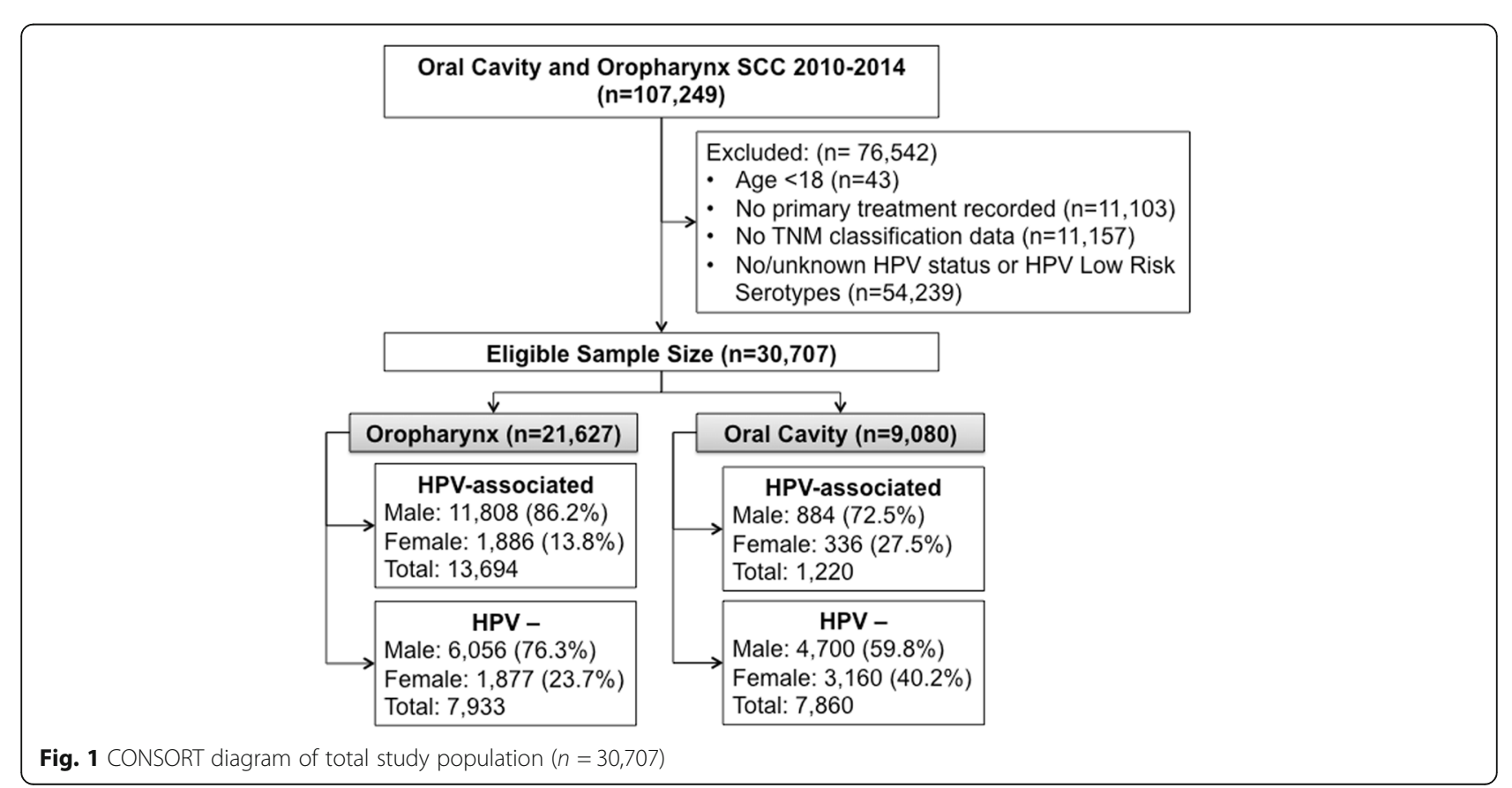


Table 1 Patient characteristics among those with oropharyngeal squamous cell carcinoma based on sex and HPV status

\begin{tabular}{|c|c|c|c|c|c|c|c|c|c|c|}
\hline & \multicolumn{5}{|c|}{ Oropharynx HPV-associated } & \multicolumn{5}{|c|}{ Oropharynx HPV - } \\
\hline & \multicolumn{2}{|l|}{ Male } & \multicolumn{2}{|c|}{ Female } & \multirow[b]{2}{*}{$p$-value } & \multicolumn{2}{|l|}{ Male } & \multicolumn{2}{|l|}{ Female } & \multirow[b]{2}{*}{$p$-value } \\
\hline & Count & $\%$ & Count & $\%$ & & Count & $\%$ & Count & $\%$ & \\
\hline Mean age (years) & 58,69 & & 59,65 & & $<0.001$ & 60,74 & & 61,66 & & $<0.001$ \\
\hline Ethnicity & & & & & 0.006 & & & & & 0.32 \\
\hline White & 10,997 & $94.0 \%$ & 1717 & $91.9 \%$ & & 5167 & $86.0 \%$ & 1566 & $84.2 \%$ & \\
\hline Black & 538 & $4.6 \%$ & 107 & $5.7 \%$ & & 709 & $11.8 \%$ & 243 & $13.1 \%$ & \\
\hline American Indian/Eskimo & 22 & $0.2 \%$ & 5 & $0.3 \%$ & & 13 & $0.2 \%$ & 7 & $0.4 \%$ & \\
\hline Asian/Pacific Islander & 103 & $0.9 \%$ & 28 & $1.5 \%$ & & 87 & $1.4 \%$ & 32 & $1.7 \%$ & \\
\hline Other & 45 & $0.4 \%$ & 12 & $0.6 \%$ & & 32 & $0.5 \%$ & 11 & $0.6 \%$ & \\
\hline Charlson/Deyo Score & & & & & $<0.001$ & & & & & 0.72 \\
\hline 0 & 9957 & $84.3 \%$ & 1530 & $81.1 \%$ & & 4817 & $79.5 \%$ & 1477 & $78.7 \%$ & \\
\hline 1 & 1486 & $12.6 \%$ & 272 & $14.4 \%$ & & 942 & $15.6 \%$ & 304 & $16.2 \%$ & \\
\hline 2 & 365 & $3.1 \%$ & 84 & $4.5 \%$ & & 297 & $4.9 \%$ & 96 & $5.1 \%$ & \\
\hline \multicolumn{11}{|l|}{ AJCC Clinical Staging } \\
\hline T Staging & & & & & $<0.001$ & & & & & 0.002 \\
\hline T0 & 85 & $0.7 \%$ & 13 & $0.7 \%$ & & 16 & $0.3 \%$ & 6 & $0.3 \%$ & \\
\hline $\mathrm{T} 1$ & 3225 & $27.4 \%$ & 582 & $31.1 \%$ & & 1221 & $20.3 \%$ & 428 & $23.0 \%$ & \\
\hline $\mathrm{T} 2$ & 4834 & $41.1 \%$ & 770 & $41.2 \%$ & & 2097 & $34.9 \%$ & 671 & $36.0 \%$ & \\
\hline T3 & 1925 & $16.4 \%$ & 254 & $13.6 \%$ & & 1319 & $22.0 \%$ & 339 & $18.2 \%$ & \\
\hline $\mathrm{T} 4$ & 1354 & $11.5 \%$ & 190 & $10.2 \%$ & & 1180 & $19.7 \%$ & 380 & $20.4 \%$ & \\
\hline TX & 326 & $2.8 \%$ & 61 & $3.3 \%$ & & 170 & $2.8 \%$ & 38 & $2.0 \%$ & \\
\hline N Staging & & & & & $<0.001$ & & & & & $<0.001$ \\
\hline No & 1336 & $11.3 \%$ & 318 & $16.9 \%$ & & 1410 & $23.4 \%$ & 594 & $31.7 \%$ & \\
\hline N1 & 1874 & $15.9 \%$ & 424 & $22.5 \%$ & & 997 & $16.5 \%$ & 344 & $18.3 \%$ & \\
\hline N2 & 8023 & $68.1 \%$ & 1088 & $57.8 \%$ & & 3298 & $54.6 \%$ & 877 & $46.8 \%$ & \\
\hline N3 & 522 & $4.4 \%$ & 49 & $2.6 \%$ & & 293 & $4.9 \%$ & 50 & $2.7 \%$ & \\
\hline$N X$ & 33 & $0.3 \%$ & 3 & $0.2 \%$ & & 37 & $0.6 \%$ & 10 & $0.5 \%$ & \\
\hline M Staging & & & & & 0.812 & & & & & 0.013 \\
\hline MO & 11,042 & $97.7 \%$ & 1763 & $98.0 \%$ & & 5435 & $95.4 \%$ & 1718 & $96.7 \%$ & \\
\hline M1 & 265 & $2.3 \%$ & 36 & $2.0 \%$ & & 264 & $4.6 \%$ & 58 & $3.3 \%$ & \\
\hline Primary Site & & & & & $<0.001$ & & & & & 0.005 \\
\hline Base of Tongue & 4845 & $41.0 \%$ & 611 & $32.4 \%$ & & 2543 & $42.0 \%$ & 745 & $39.7 \%$ & \\
\hline Tonsil & 6258 & $53.0 \%$ & 1150 & $61.0 \%$ & & 2615 & $43.2 \%$ & 796 & $42.4 \%$ & \\
\hline Other OP & 705 & $6.0 \%$ & 125 & $6.6 \%$ & & 898 & $14.8 \%$ & 336 & $17.9 \%$ & \\
\hline Insurance Status & & & & & $<0.001$ & & & & & $<0.001$ \\
\hline Not Insured & 437 & $3.7 \%$ & 71 & $3.8 \%$ & & 383 & $6.4 \%$ & 111 & $6.0 \%$ & \\
\hline Private Insurance/Managed Care & 7264 & $62.2 \%$ & 989 & $52.9 \%$ & & 2601 & $43.8 \%$ & 732 & $39.5 \%$ & \\
\hline Medicaid & 753 & $6.4 \%$ & 162 & $8.7 \%$ & & 703 & $11.8 \%$ & 240 & $13.0 \%$ & \\
\hline Medicare & 2940 & $25.2 \%$ & 634 & $33.9 \%$ & & 2121 & $35.7 \%$ & 750 & $40.5 \%$ & \\
\hline Other Government & 290 & $2.5 \%$ & 12 & $0.6 \%$ & & 132 & $2.2 \%$ & 18 & $1.0 \%$ & \\
\hline Median Income Quartiles 2008-2012 & & & & & 0.002 & & & & & 0.043 \\
\hline$<\$ 38,000$ & 1472 & $12.5 \%$ & 274 & $14.6 \%$ & & 1184 & $19.7 \%$ & 389 & $20.9 \%$ & \\
\hline$\$ 38,000-\$ 47,999$ & 2443 & $20.8 \%$ & 435 & $23.1 \%$ & & 1335 & $22.2 \%$ & 451 & $24.2 \%$ & \\
\hline
\end{tabular}


Table 1 Patient characteristics among those with oropharyngeal squamous cell carcinoma based on sex and HPV status (Continued)

\begin{tabular}{|c|c|c|c|c|c|c|c|c|c|c|}
\hline & \multicolumn{5}{|c|}{ Oropharynx HPV-associated } & \multicolumn{5}{|c|}{ Oropharynx HPV - } \\
\hline & \multicolumn{2}{|l|}{ Male } & \multicolumn{2}{|l|}{ Female } & \multirow[b]{2}{*}{$p$-value } & \multicolumn{2}{|l|}{$\overline{\text { Male }}$} & \multicolumn{2}{|c|}{ Female } & \multirow[b]{2}{*}{$p$-value } \\
\hline & Count & $\%$ & Count & $\%$ & & Count & $\%$ & Count & $\%$ & \\
\hline$\$ 48,000-\$ 62,999$ & 3265 & $27.7 \%$ & 488 & $25.9 \%$ & & 1606 & $26.7 \%$ & 491 & $26.4 \%$ & \\
\hline$\$ 63,000+$ & 4588 & $39.0 \%$ & 685 & $36.4 \%$ & & 1900 & $31.5 \%$ & 530 & $28.5 \%$ & \\
\hline Urban/Rural 2013 & & & & & 0.190 & & & & & 0.27 \\
\hline Metro & 9826 & $85.3 \%$ & 1573 & $84.8 \%$ & & 5053 & $85.5 \%$ & 1539 & $84.0 \%$ & \\
\hline Urban & 1488 & $12.9 \%$ & 259 & $14.0 \%$ & & 775 & $13.1 \%$ & 265 & $14.5 \%$ & \\
\hline Rural & 200 & $1.7 \%$ & 24 & $1.3 \%$ & & 80 & $1.4 \%$ & 28 & $1.5 \%$ & \\
\hline Facility Type & & & & & 0.214 & & & & & 0.346 \\
\hline Community Cancer Program & 743 & $6.4 \%$ & 128 & $7.0 \%$ & & 531 & $8.9 \%$ & 159 & $8.6 \%$ & \\
\hline Comprehensive Community Cancer Program & 3719 & $31.8 \%$ & 596 & $32.7 \%$ & & 2153 & $36.0 \%$ & 632 & $34.3 \%$ & \\
\hline Academic/Research Program & 5810 & $49.7 \%$ & 907 & $49.8 \%$ & & 2618 & $43.8 \%$ & 851 & $46.2 \%$ & \\
\hline Integrated Network Cancer Program & 1407 & $12.0 \%$ & 192 & $10.5 \%$ & & 671 & $11.2 \%$ & 199 & $10.8 \%$ & \\
\hline Other specified types of cancer programs & 0 & $0.0 \%$ & 0 & $0.0 \%$ & & 0 & $0.0 \%$ & 0 & $0.0 \%$ & \\
\hline Facility Location & & & & & 0.110 & & & & & 0.130 \\
\hline East & 2440 & $20.9 \%$ & 419 & $23.0 \%$ & & 1223 & $20.5 \%$ & 398 & $21.6 \%$ & \\
\hline South & 3915 & $33.5 \%$ & 610 & $33.5 \%$ & & 2454 & $41.1 \%$ & 704 & $38.2 \%$ & \\
\hline Midwest & 3196 & $27.4 \%$ & 493 & $27.0 \%$ & & 1408 & $23.6 \%$ & 467 & $25.4 \%$ & \\
\hline West & 2128 & $18.2 \%$ & 301 & $16.5 \%$ & & 888 & $14.9 \%$ & 272 & $14.8 \%$ & \\
\hline Treatment Group & & & & & $<0.001$ & & & & & $<0.001$ \\
\hline No treatment & 210 & $1.8 \%$ & 31 & $1.6 \%$ & & 286 & $4.7 \%$ & 90 & $4.8 \%$ & \\
\hline Radiation only & 868 & $7.4 \%$ & 158 & $8.4 \%$ & & 508 & $8.4 \%$ & 186 & $9.9 \%$ & \\
\hline Radiation and Chemo & 7185 & $60.8 \%$ & 1011 & $53.6 \%$ & & 3571 & $59.0 \%$ & 1004 & $53.5 \%$ & \\
\hline Surgery and Radiation & 726 & $6.1 \%$ & 155 & $8.2 \%$ & & 240 & $4.0 \%$ & 94 & $5.0 \%$ & \\
\hline Surgery, Chemotherapy and Radiation & 2027 & $17.2 \%$ & 341 & $18.1 \%$ & & 725 & $12.0 \%$ & 210 & $11.2 \%$ & \\
\hline Surgery only & 572 & $4.8 \%$ & 155 & $8.2 \%$ & & 464 & $7.7 \%$ & 218 & $11.6 \%$ & \\
\hline Chemotherapy Only & 220 & $1.9 \%$ & 35 & $1.9 \%$ & & 262 & $4.3 \%$ & 75 & $4.0 \%$ & \\
\hline
\end{tabular}

patient's primary country of residence). Patients were excluded if they were younger than 18 years old, if TNM classification or primary treatment type was unknown. Primary treatment type was classified into the following groups: no treatment, radiation only, chemoradiation therapy, surgery and radiation, surgery and chemoradiation, surgery only and chemotherapy only.

\section{Statistical analysis}

Data analyses were performed using SPSS 19.0 (IBM Corp., Armonk, NY). The comparison of mean age at diagnosis was analyzed using the Student's t-test. Proportional distribution of race, Charleson/Deyo score, primary tumor site, $\mathrm{T}$ and $\mathrm{N}$ classification, lymph node metastasis, primary treatment type, insurance status, median income quartiles, treatment facility type and location, and rural/urban classification of patient's primary country of residence were compared using chi-squared tests. Survival analysis was performed using Kaplan-Meier analysis. The comparison of survival rates among the groups was performed using the two-tailed log-rank test. The average follow up time for survival analysis in the dataset was 31.7 months. Cox proportional hazards regression model was used for multivariable survival analysis. Age, sex, race, Charleson/Deyo score (for OPSCCs only), $\mathrm{T}$ and $\mathrm{N}$ classification, site of primary tumor (for OPSCCs only), primary treatment type, insurance status and median income were entered a priori into the model. A two-sided $p$-value $<0.05$ was considered statistically significant.

Our study is exempt from review by the Yale Human Research Protection Program because it uses a pre-existing, de-identified public database.

\section{Results}

Our study population $(n=30,707)$ included 13,694 OP HPV-associated; 7933 OP HPV- cancers; 1220 OC HPV-associated and 7860 OC HPV- cancers (Fig. 1). 
Table 2 Patient characteristics among those with oral cavity squamous cell carcinoma based on sex and HPV status

\begin{tabular}{|c|c|c|c|c|c|c|c|c|c|c|}
\hline & \multicolumn{5}{|c|}{ Oral Cavity HPV-associated } & \multicolumn{5}{|c|}{ Oral Cavity HPV - } \\
\hline & \multicolumn{2}{|l|}{ Male } & \multicolumn{2}{|l|}{ Female } & \multirow[b]{2}{*}{$p$-value } & \multicolumn{2}{|l|}{ Male } & \multicolumn{2}{|c|}{ Female } & \multirow[b]{2}{*}{$p$-value } \\
\hline & Count & $\%$ & Count & $\%$ & & Count & $\%$ & Count & $\%$ & \\
\hline Mean age (years) & 58,85 & & 59,72 & & $<0.001$ & 61,35 & & 63,95 & & $<0.001$ \\
\hline Ethnicity & & & & & 0.502 & & & & & 0.512 \\
\hline White & 804 & $91.6 \%$ & 296 & $88.9 \%$ & & 4093 & $87.7 \%$ & 2777 & $88.7 \%$ & \\
\hline Black & 44 & $5.0 \%$ & 25 & $7.5 \%$ & & 371 & $7.9 \%$ & 218 & $7.0 \%$ & \\
\hline American Indian/Eskimo & 2 & $0.2 \%$ & 1 & $0.3 \%$ & & 15 & $0.3 \%$ & 7 & $0.2 \%$ & \\
\hline Asian/Pacific Islander & 20 & $2.3 \%$ & 9 & $2.7 \%$ & & 151 & $3.2 \%$ & 103 & $3.3 \%$ & \\
\hline Other & 8 & $0.9 \%$ & 2 & $0.6 \%$ & & 39 & $0.8 \%$ & 26 & $0.8 \%$ & \\
\hline Charlson/Deyo Score & & & & & 0.11 & & & & & 0.92 \\
\hline 0 & 689 & $77.9 \%$ & 259 & $77.1 \%$ & & 3607 & $76.7 \%$ & 2434 & $77.0 \%$ & \\
\hline 1 & 165 & $18.7 \%$ & 57 & $17.0 \%$ & & 837 & $17.8 \%$ & 552 & $17.5 \%$ & \\
\hline 2 & 30 & $3.4 \%$ & 20 & $6.0 \%$ & & 256 & $5.4 \%$ & 174 & $5.5 \%$ & \\
\hline \multicolumn{11}{|l|}{ AJCC Clinical Staging } \\
\hline T Staging & & & & & 0.005 & & & & & $<0.001$ \\
\hline TO & 4 & $0.5 \%$ & 0 & $0.0 \%$ & & 8 & $0.2 \%$ & 5 & $0.2 \%$ & \\
\hline $\mathrm{T} 1$ & 251 & $29.3 \%$ & 129 & $40.1 \%$ & & 1579 & $34.6 \%$ & 1292 & $42.3 \%$ & \\
\hline $\mathrm{T} 2$ & 277 & $32.4 \%$ & 101 & $31.4 \%$ & & 1409 & $30.8 \%$ & 914 & $29.9 \%$ & \\
\hline T3 & 96 & $11.2 \%$ & 32 & $9.9 \%$ & & 506 & $11.1 \%$ & 271 & $8.9 \%$ & \\
\hline T4 & 216 & $25.2 \%$ & 56 & $17.4 \%$ & & 1039 & $22.7 \%$ & 551 & $18.1 \%$ & \\
\hline TX & 12 & $1.4 \%$ & 4 & $1.2 \%$ & & 29 & $0.6 \%$ & 19 & $0.6 \%$ & \\
\hline N Staging & & & & & 0.003 & & & & & $<0.001$ \\
\hline NO & 420 & $47.5 \%$ & 198 & $59.5 \%$ & & 3001 & $64.0 \%$ & 2226 & $70.7 \%$ & \\
\hline N1 & 130 & $14.7 \%$ & 46 & $13.8 \%$ & & 552 & $11.8 \%$ & 332 & $10.5 \%$ & \\
\hline N2 & 313 & $35.4 \%$ & 86 & $25.8 \%$ & & 1038 & $22.2 \%$ & 551 & $17.5 \%$ & \\
\hline N3 & 15 & $1.7 \%$ & 2 & $0.6 \%$ & & 57 & $1.2 \%$ & 14 & $0.4 \%$ & \\
\hline NX & 6 & $0.7 \%$ & 1 & $0.3 \%$ & & 38 & $0.8 \%$ & 27 & $0.9 \%$ & \\
\hline M Staging & & & & & 0.939 & & & & & 0.004 \\
\hline Mo & 833 & $97.9 \%$ & 313 & $97.8 \%$ & & 4339 & $97.7 \%$ & 2959 & $98.6 \%$ & \\
\hline M1 & 18 & $2.1 \%$ & 7 & $2.2 \%$ & & 102 & $2.3 \%$ & 41 & $1.4 \%$ & \\
\hline Insurance Status & & & & & 0.09 & & & & & $<0.001$ \\
\hline Not Insured & 51 & $5.8 \%$ & 23 & $6.9 \%$ & & 236 & $5.1 \%$ & 132 & $4.2 \%$ & \\
\hline Private Insurance/Managed Care & 420 & $48.1 \%$ & 145 & $43.5 \%$ & & 1935 & $41.9 \%$ & 1197 & $38.5 \%$ & \\
\hline Medicaid & 90 & $10.3 \%$ & 30 & $9.0 \%$ & & 515 & $11.1 \%$ & 260 & $8.4 \%$ & \\
\hline Medicare & 286 & $32.8 \%$ & 131 & $39.3 \%$ & & 1850 & $40.0 \%$ & 1480 & $47.6 \%$ & \\
\hline Other Government & 26 & $3.0 \%$ & 4 & $1.2 \%$ & & 85 & $1.8 \%$ & 38 & $1.2 \%$ & \\
\hline Median Income Quartiles 2008-2012 & & & & & 0.22 & & & & & 0.010 \\
\hline$<\$ 38,000$ & 137 & $15.6 \%$ & 47 & $14.0 \%$ & & 843 & $18.0 \%$ & 512 & $16.3 \%$ & \\
\hline$\$ 38,000-\$ 47,999$ & 206 & $23.4 \%$ & 93 & $27.8 \%$ & & 1171 & $25.0 \%$ & 725 & $23.0 \%$ & \\
\hline$\$ 48,000-\$ 62,999$ & 264 & $30.0 \%$ & 85 & $25.4 \%$ & & 1250 & $26.7 \%$ & 863 & $27.4 \%$ & \\
\hline$\$ 63,000+$ & 272 & $30.9 \%$ & 110 & $32.8 \%$ & & 1426 & $30.4 \%$ & 1046 & $33.2 \%$ & \\
\hline Urban/Rural 2013 & & & & & 0.510 & & & & & 0.072 \\
\hline Metro & 741 & $85.2 \%$ & 287 & $87.8 \%$ & & 3799 & $82.8 \%$ & 2579 & $83.9 \%$ & \\
\hline
\end{tabular}


Table 2 Patient characteristics among those with oral cavity squamous cell carcinoma based on sex and HPV status (Continued)

\begin{tabular}{|c|c|c|c|c|c|c|c|c|c|c|}
\hline & \multicolumn{5}{|c|}{ Oral Cavity HPV-associated } & \multicolumn{5}{|c|}{ Oral Cavity HPV - } \\
\hline & \multicolumn{2}{|l|}{ Male } & \multicolumn{2}{|l|}{ Female } & \multirow[b]{2}{*}{$p$-value } & \multicolumn{2}{|l|}{ Male } & \multicolumn{2}{|l|}{ Female } & \multirow[b]{2}{*}{$p$-value } \\
\hline & Count & $\%$ & Count & $\%$ & & Count & $\%$ & Count & $\%$ & \\
\hline Urban & 114 & $13.1 \%$ & 35 & $10.7 \%$ & & 708 & $15.4 \%$ & 461 & $15.0 \%$ & \\
\hline Rural & 15 & $1.7 \%$ & 5 & $1.5 \%$ & & 81 & $1.8 \%$ & 35 & $1.1 \%$ & \\
\hline Facility Type & & & & & 0.507 & & & & & 0.967 \\
\hline Community Cancer Program & 64 & $7.6 \%$ & 20 & $6.6 \%$ & & 274 & $6.1 \%$ & 188 & $6.3 \%$ & \\
\hline Comprehensive Community Cancer Program & 239 & $28.3 \%$ & 75 & $24.8 \%$ & & 1226 & $27.3 \%$ & 823 & $27.6 \%$ & \\
\hline Academic/Research Program & 466 & $55.2 \%$ & 176 & $58.1 \%$ & & 2487 & $55.3 \%$ & 1643 & $55.0 \%$ & \\
\hline Integrated Network Cancer Program & 75 & $8.9 \%$ & 32 & $10.6 \%$ & & 507 & $11.3 \%$ & 331 & $11.1 \%$ & \\
\hline Other specified types of cancer programs & 0 & $0.0 \%$ & 0 & $0.0 \%$ & & 0 & $0.0 \%$ & 0 & $0.0 \%$ & \\
\hline Facility Location & & & & & 0.990 & & & & & 0.026 \\
\hline East & 178 & $21.1 \%$ & 64 & $21.1 \%$ & & 995 & $22.1 \%$ & 662 & $22.2 \%$ & \\
\hline South & 278 & $32.9 \%$ & 98 & $32.3 \%$ & & 1660 & $36.9 \%$ & 1014 & $34.0 \%$ & \\
\hline Midwest & 240 & $28.4 \%$ & 87 & $28.7 \%$ & & 1149 & $25.6 \%$ & 793 & $26.6 \%$ & \\
\hline West & 148 & $17.5 \%$ & 54 & $17.8 \%$ & & 690 & $15.4 \%$ & 516 & $17.3 \%$ & \\
\hline Treatment Group & & & & & $<0.001$ & & & & & $<0.001$ \\
\hline No treatment & 29 & $3.3 \%$ & 11 & $3.3 \%$ & & 163 & $3.5 \%$ & 120 & $3.8 \%$ & \\
\hline Radiation only & 56 & $6.3 \%$ & 26 & $7.7 \%$ & & 282 & $6.0 \%$ & 217 & $6.9 \%$ & \\
\hline Radiation and Chemo & 269 & $30.4 \%$ & 62 & $18.5 \%$ & & 717 & $15.3 \%$ & 356 & $11.3 \%$ & \\
\hline Surgery and Radiation & 100 & $11.3 \%$ & 37 & $11.0 \%$ & & 573 & $12.2 \%$ & 396 & $12.5 \%$ & \\
\hline Surgery, Chemotherapy and Radiation & 146 & $16.5 \%$ & 55 & $16.4 \%$ & & 806 & $17.1 \%$ & 408 & $12.9 \%$ & \\
\hline Surgery only & 264 & $29.9 \%$ & 139 & $41.4 \%$ & & 2072 & $44.1 \%$ & 1620 & $51.3 \%$ & \\
\hline Chemotherapy Only & 20 & $2.3 \%$ & 6 & $1.8 \%$ & & 87 & $1.9 \%$ & 43 & $1.4 \%$ & \\
\hline
\end{tabular}

The presence of HPV was correlated with higher proportion of disease burden among men. Among the OP HPV-associated and HPV- cohorts, 86.2 and $76.3 \%$ of patients were men respectively. Among the OC HPV-associated and HPV- cohorts, 76.3 and $59.8 \%$ were men respectively. Each group was further analyzed for baseline characteristic differences by sex (Tables 1 and 2).

\section{Baseline characteristic differences}

Within all four groups, women were on average older at age of diagnosis ( $p<0.001$ for each group). Women were generally diagnosed with cancers in earlier $\mathrm{T}$ and $\mathrm{N}$ clinical classification than men. In OP, this difference was most pronounced in $\mathrm{N}$ classification; in OP HPV-associated cancers, $39.4 \%$ women vs. $27.2 \%$ men had N0-1 cancers ( $p<0.001)$, in OP HPV- cancers, $50.0 \%$ women vs. $39.9 \%$ men had N0-1 cancers $(p<0.001)$. In OC HPV-associated cancers, 40.1\% women had T0-1 cancers vs. $29.8 \%$ men and in OC HPV- cancers $(p=0.005), 42.3 \%$ vs. $34.8 \%$ in women and men respectively $(p<0.001)$. Women in all four groups were more likely to be treated with a modality including surgery (surgery only, surgery and radiation, or surgery and chemo-radiation; $\mathrm{p}<0.001$ in each group). For insurance coverage, more women were covered by Medicare than men across all four study populations.

\section{Factors associated with survival in OPSCCs}

Kaplan-Meier survival analysis showed no difference in OS between the two sexes in OP HPV-associated cancers $(p=0.64$; Figs. $2 \mathrm{a})$. On multivariate analysis, after accounting for age at diagnosis, ethnicity, clinical $\mathrm{T}$ and $\mathrm{N}$ classification, primary disease site, primary treatment, insurance status and median income, female sex (HR: 0.93; 95\% CI $0.79-1.009, p=0.412$ ) did not prove to be an independent prognostic factor for OS.

In OP HPV- cancers, men had a statistically significant better OS than women on Kaplan Meier survival analysis ( $p=0.035$, Fig. 2b). In multivariate analysis, female sex (HR: 1.15 ; 95\% CI 1.04-1.28, $p=0.004$ ) continued to be an independent prognostic factor for worse OS in OP HPV- cancers even after controlling for other variables (as described previously, Table 3).

The hazard of death was notably higher for both OP HPV-associated and HPV- cohorts with increasing age, higher $\mathrm{T}$ and $\mathrm{N}$ classification, cancers at sites other than base of tongue or tonsils and patients with no primary treatment (Table 3). 

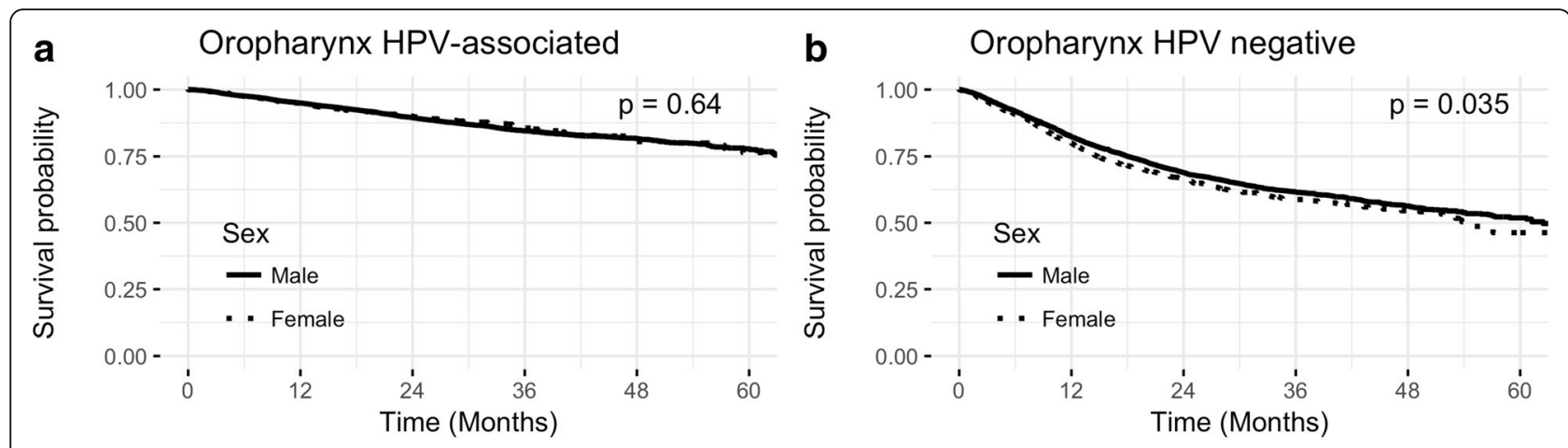

Number at risk

Number at risk
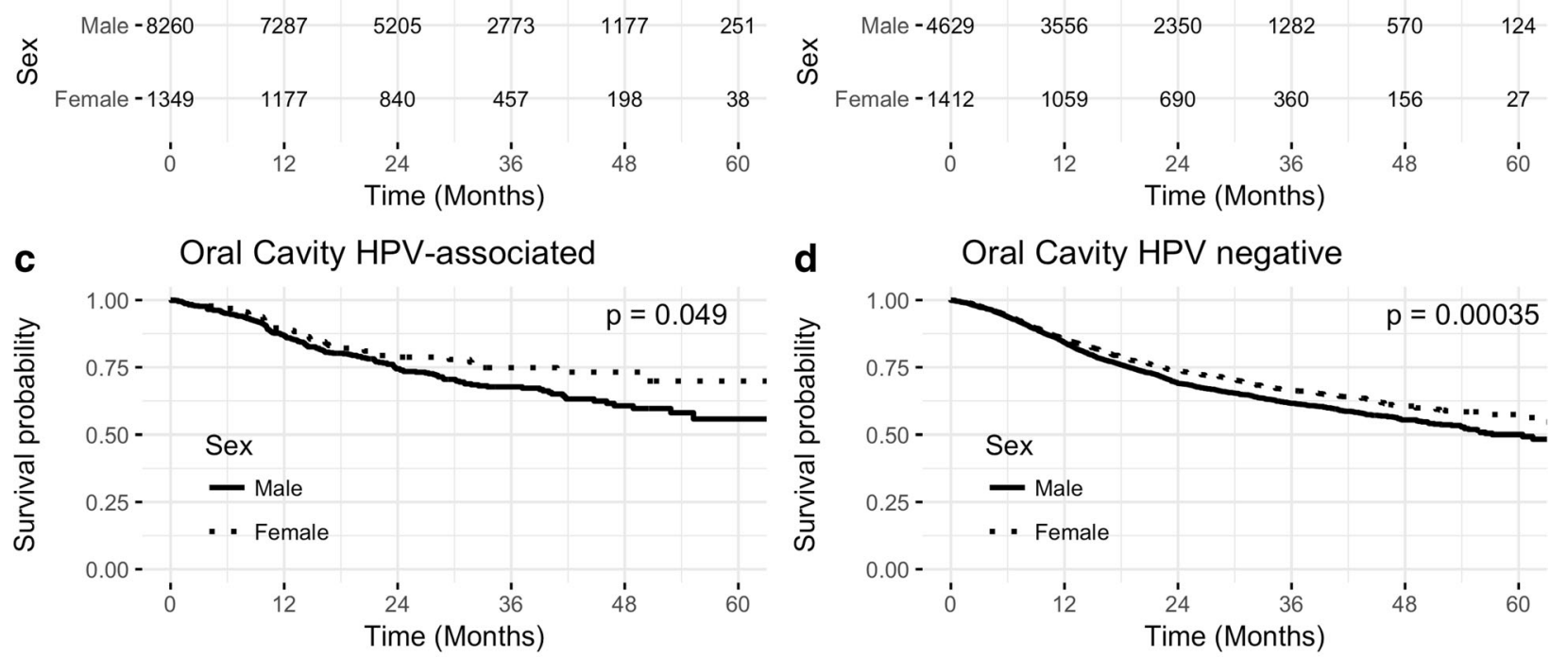

Number at risk

Number at risk
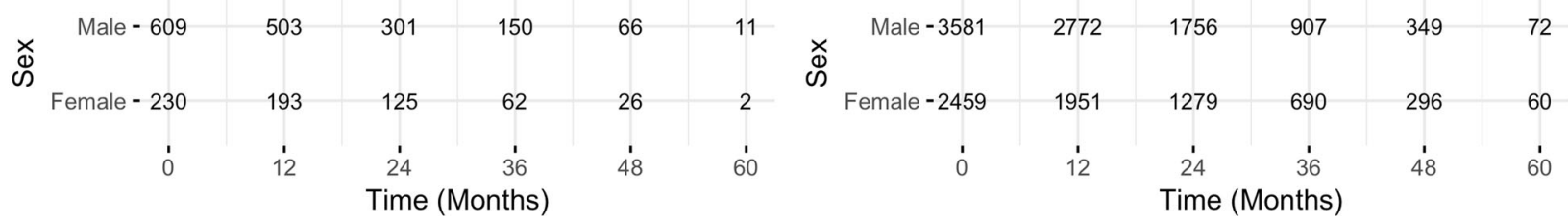

Fig. 2 Kaplan-Meier survival and number at risk a OP HPV-associated: $p=0.638, \mathbf{b}$ OP HPV negative: $p=0.035, \mathbf{c}$ OC HPV-associated: $p=0.049$, d OC HPV negative: $p<0.001$

\section{Factors associated with survival in OCSCCs}

Kaplan-Meier survival analysis showed that among OC cancers, women had better OS than men in both HPV-associated and HPV- cancers $(p=0.049, p<0.001$ respectively, Fig. 2c, d).

In contrast to the varying prognostic roles of female sex in OPSCCs, in OCSCCs, female sex remained a strong prognostic factor for better OS in both HPV-associated and HPV- cancers (HPV-associated: HR: 0.71; 95\% CI 0.0.50-0.99, $p=0.048$; HPV-: HR: 0.87; 95\% CI 0.78-0.95, $p=0.004$; Table 4) after controlling for over variables. In OC HPV-associated cancers, age (HR: 1.02; 95\% CI 1.00$1.04, p=0.01$ ) and black race (HR: $1.88 ; 95 \%$ CI 1.14-3.11, $p=0.013)$ were significant predictors of OS in patients. In OC HPV- cancers, age (HR: 1.02; 95\% CI 1.02-1.02, $p<0.001), \mathrm{N}$ classification $(p<0.001)$ and having higher median income $\$ 63,000+((\mathrm{HR}: 0.77 ; 95 \% \mathrm{CI}$ $0.67-0.88, p<0.001$ ), and having treatment (over no treatment; $p<0.001$ for all except chemotherapy only group $p=0.31$ ) were all significant predictors of OS.

\section{Discussion}

HPV status and its importance as a prognostic marker in oropharyngeal SCCs has been well established [13, 14]. The prognostic associations of HPV status with other clinical factors such as sex and primary tumor 
Table 3 Cox proportional hazards regression analysis for patients with oropharyngeal squamous cell carcinoma

\begin{tabular}{|c|c|c|c|c|}
\hline & \multicolumn{2}{|c|}{ Oropharynx HPV-associated } & \multicolumn{2}{|l|}{ Oropharynx HPV - } \\
\hline & $\mathrm{HR}(95 \% \mathrm{Cl})$ & $P$ & $\mathrm{HR}(95 \% \mathrm{Cl})$ & $P$ \\
\hline Mean age & $1.02(1.01-1.03)$ & $<0.001$ & $1.01(1.00-1.02)$ & $<0.001$ \\
\hline \multicolumn{5}{|l|}{ Ethnicity } \\
\hline White & 1.00 & & 1.00 & \\
\hline Black & $0.86(0.67-1.10)$ & 0.25 & $1.14(1.01-1.30)$ & 0.03 \\
\hline American Indian/Eskimo & $0.61(0.15-2.47)$ & 0.49 & $0.22(0.03-1.58)$ & 0.13 \\
\hline Asian/Pacific Islander & $0.52(0.24-1.10)$ & 0.09 & $0.75(0.50-1.14)$ & 0.19 \\
\hline Other & $0.63(0.15-2.52)$ & 0.51 & $1.29(0.67-2.49)$ & 0.44 \\
\hline \multicolumn{5}{|l|}{ Sex } \\
\hline Men & 1.00 & & 1.00 & \\
\hline Women & $0.93(0.79-1.09)$ & 0.412 & $1.15(1.04-1.28)$ & 0.004 \\
\hline \multicolumn{5}{|l|}{ Charlson/Deyo Score } \\
\hline 0 & 1.0 & & 1.0 & \\
\hline 1 & $1.42(1.23-1.65)$ & $<0.001$ & $1.31(1.17-1.46)$ & $<0.001$ \\
\hline 2 & $1.97(1.56-2.48)$ & $<0.001$ & $1.49(1.25-1.77)$ & $<0.001$ \\
\hline \multicolumn{5}{|l|}{ AJCC Clinical Staging } \\
\hline \multicolumn{5}{|l|}{ T Staging } \\
\hline T0 & 1.00 & & 1.00 & \\
\hline $\mathrm{T} 1$ & $2.61(0.64-10.5)$ & 0.18 & $1.31(0.32-5.30)$ & 0.70 \\
\hline $\mathrm{T} 2$ & $4.24(1.05-17.0)$ & 0.04 & $1.93(0.48-7.79)$ & 0.35 \\
\hline $\mathrm{T} 3$ & $6.47(1.60-26.1)$ & 0.01 & $3.26(0.81-13.1)$ & 0.10 \\
\hline $\mathrm{T} 4$ & $9.92(2.45-40.0)$ & 0.00 & $4.35(1.08-17.5)$ & 0.04 \\
\hline TX & $3.93(0.93-16.4)$ & 0.06 & $2.65(0.64-10.9)$ & 0.18 \\
\hline \multicolumn{5}{|l|}{ N Staging } \\
\hline No & 1.00 & & 1.00 & \\
\hline N1 & $0.81(0.64-1.01)$ & 0.07 & $0.95(0.82-1.10)$ & 0.53 \\
\hline N2 & $1.14(0.95-1.37)$ & 0.14 & $1.10(0.98-1.24)$ & 0.09 \\
\hline N3 & $2.06(1.58-2.67)$ & $<0.001$ & $1.76(1.45-2.15)$ & $<0.001$ \\
\hline NX & $0.73(0.29-1.83)$ & 0.51 & $1.47(0.91-2.36)$ & 0.11 \\
\hline \multicolumn{5}{|l|}{ Primary Site } \\
\hline Base of Tongue & 1.00 & & 1.00 & \\
\hline Tonsil & $1.03(0.91-1.16)$ & 0.63 & $0.87(0.79-0.96)$ & 0.01 \\
\hline Other OP & $1.48(1.21-1.81)$ & $<0.001$ & $1.15(1.02-1.30)$ & 0.02 \\
\hline \multicolumn{5}{|l|}{ Insurance Status } \\
\hline Not Insured & 1.00 & & 1.00 & \\
\hline Private Insurance/Managed Care & $0.53(0.41-0.68)$ & $<0.001$ & $0.61(0.51-0.72)$ & $<0.001$ \\
\hline Medicaid & $1.04(0.78-1.38)$ & 0.77 & $1.11(0.93-1.34)$ & 0.23 \\
\hline Medicare & $0.99(0.76-1.30)$ & 0.98 & $0.94(0.78-1.13)$ & 0.55 \\
\hline Other Government & $0.96(0.63-1.46)$ & 0.85 & $0.96(0.67-1.36)$ & 0.83 \\
\hline \multicolumn{5}{|l|}{ Median Income Quartiles 2008-2012 } \\
\hline$<\$ 38,000$ & 1.00 & & 1.00 & \\
\hline$\$ 38,000-\$ 47,999$ & $0.89(0.75-1.06)$ & 0.21 & $0.9(0.79-1.02)$ & 0.11 \\
\hline$\$ 48,000-\$ 62,999$ & $0.78(0.65-0.93)$ & 0.01 & $0.83(0.73-0.94)$ & 0.01 \\
\hline$\$ 63,000+$ & $0.65(0.54-0.77)$ & $<0.001$ & $0.73(0.64-0.83)$ & $<0.001$ \\
\hline
\end{tabular}


Table 3 Cox proportional hazards regression analysis for patients with oropharyngeal squamous cell carcinoma (Continued)

\begin{tabular}{|c|c|c|c|c|}
\hline & \multicolumn{2}{|c|}{ Oropharynx HPV-associated } & \multicolumn{2}{|c|}{ Oropharynx HPV - } \\
\hline & $\overline{\mathrm{HR}(95 \% \mathrm{Cl})}$ & $P$ & $\mathrm{HR}(95 \% \mathrm{Cl})$ & $P$ \\
\hline \multicolumn{5}{|l|}{ Treatment Group } \\
\hline No treatment & 1.00 & & 1.00 & \\
\hline Radiation only & $0.34(0.24-0.48)$ & $<0.001$ & $0.44(0.35-0.54)$ & $<0.001$ \\
\hline Radiation and Chemo & $0.22(0.16-0.29)$ & $<0.001$ & $0.27(0.23-0.33)$ & $<0.001$ \\
\hline Surgery and Radiation & $0.16(0.10-0.24)$ & $<0.001$ & $0.20(0.14-0.28)$ & $<0.001$ \\
\hline Surgery, Chemotherapy and Radiation & $0.21(0.15-0.29)$ & $<0.001$ & $0.29(0.23-0.35)$ & $<0.001$ \\
\hline Surgery only & $0.21(0.14-0.32)$ & $<0.001$ & $0.37(0.29-0.47)$ & $<0.001$ \\
\hline Chemotherapy Only & $1.08(0.76-1.54)$ & 0.64 & $0.94(0.76-1.18)$ & 0.64 \\
\hline
\end{tabular}

location have not been well investigated. Given that HNSCCs affect the two sexes disproportionately $(80 \%$ men), we hypothesized that sex will be a prognostic factor for survival in HNSCCs. Our study found that sex does appear to play a distinct role in predicting OS and that the prognostic value of sex is dependent on HPV status and location of primary tumor. This finding is consistent with the idea that HPV-driven cancers in non-OP locations exhibit distinct clinical behavior and possess unique risk factors than HPV-driven cancers in OP $[8,9]$.

Molecular underpinnings of the HPV infection between the two sexes also vary. One Finnish study examining the clearance of HPV DNA using oral rinses between spouses found earlier virus clearance in men than in women as well as significantly different cumulative clearance rates ( $5 \%$ vs. $0 \%$ clearance in men and women respectively over 24 months) [15]. In a long-term prospective 6 year study of asymptomatic HPV infections, Syrjänen and colleagues found a 5.5 fold number of viral HPV copies in women than in men who were able to clear the infection [16]. Although similar copy numbers were found between sexes for those with persistent infections, 71\% of the HPV DNA was integrated or mixed in women vs. $57 \%$ in men. Full integration of the HPV episome into human chromosomes has been shown to be an early event in cervical carcinogenesis [17, 18], though its role in oral mucosal carcinogenesis is still debated. Nonetheless, these studies reflect a distinction in HPV's molecular behavior between sexes that needs to be further categorized.

Prior studies have been inconclusive on the significance of sex as a prognostic marker for overall survival. A recent two-institution retrospective study found sex to be prognostic in OPSCCs even after accounting for HPV-status [9]. The authors examined 860 patients with OPSCCs (including HPV-associated and HPV- patients) and performed a multivariate regression model. Our study utilizes more targeted patient subgroups that specifically examines the role of sex among HPV-associated or HPV- patients. To our knowledge, our study is the largest study with patients and their HPV status spanning across the entire U.S. As a result, our sample provides the power for the subgroup analyses for the detection of differences in sex. However, due to the nature of the national cancer registry, there is inherent uncertainty to the nature of our data as the quality of the data relies on the accuracy of data entry, diagnosis and treatment at over 1500 hospitals. In comparison, Fakhry et al.'s two-institution study limits their data inaccuracies due to a smaller sample size.

Existing research has shown that women have a significant survival benefit in many cancers outside of the head and neck region [19]. However, for HPVOPSCCs, we found the opposite where men have better survival than women. This similar trend also exists in patients with bladder cancer $[20,21]$. The reason for this observed survival advantage is unknown. Preclinical studies support a role for sex hormones as cofactors for HPV-related malignancies [22, 23] though other unidentified factors may also be responsible for this unique sex-specific finding. One study found the progesterone antagonists and nuclease-resistant oligomers containing HPV-16 response element are able to abrogate cell growth and E6/E7 gene transcription [22]. Another study examining HPV-induced laryngeal tumors found estradiol stimulated proliferation while 2-hydroxyestrone was anti-proliferative [23]. Both preclinical studies found hormonal interactions using HPV-associated tumor models, thus this does not fully explain our findings in the HPV- OPSCC cohort. Perhaps there exists an interaction between HPV and sex hormones in the OP sub-site, which improves the survival of women thus equalizing overall survival between the two sexes. Nonetheless, we acknowledge the proximity of the Kaplan-Meier survival curve between the two sexes in the HPV- OPSCC cohort. Given the absence of tobacco and alcohol data, it is possible that the two sexes may have no survival difference in HPV- OPSCCs. 
Table 4 Cox proportional hazards regression analysis for patients with oral cavity squamous cell carcinoma

\begin{tabular}{|c|c|c|c|c|}
\hline & \multicolumn{2}{|c|}{ Oral Cavity HPV-associated } & \multicolumn{2}{|l|}{ Oral Cavity HPV - } \\
\hline & $\mathrm{HR}(95 \% \mathrm{Cl})$ & $P$ & $\mathrm{HR}(95 \% \mathrm{Cl})$ & $P$ \\
\hline Mean age & $1.02(1.00-1.04)$ & 0.010 & $1.02(1.02-1.02)$ & $<0.001$ \\
\hline \multicolumn{5}{|l|}{ Ethnicity } \\
\hline White & 1.00 & & 1.00 & \\
\hline Black & $1.88(1.14-3.11)$ & 0.013 & $0.93(0.79-1.09)$ & 0.41 \\
\hline American Indian/Eskimo & a & & $1.18(0.52-2.64)$ & 0.68 \\
\hline Asian/Pacific Islander & $1.60(0.64-4.00)$ & 0.312 & $0.93(0.71-1.22)$ & 0.63 \\
\hline Other & $0.65(0.09-4.78)$ & 0.678 & $0.54(0.30-0.99)$ & 0.05 \\
\hline \multicolumn{5}{|l|}{ Sex } \\
\hline Men & 1.00 & & 1.00 & \\
\hline Women & $0.71(0.50-0.99)$ & 0.048 & $0.87(0.78-0.95)$ & 0.004 \\
\hline \multicolumn{5}{|l|}{ AJCC Clinical Staging } \\
\hline \multicolumn{5}{|l|}{ T Staging } \\
\hline TO & 1.00 & & 1.00 & \\
\hline $\mathrm{T} 1$ & $0.36(0.07-1.65)$ & 0.189 & $0.45(0.14-1.43)$ & 0.18 \\
\hline $\mathrm{T} 2$ & $0.61(0.13-2.80)$ & 0.529 & $0.79(0.25-2.49)$ & 0.70 \\
\hline T3 & $0.90(0.19-4.21)$ & 0.903 & $1.07(0.34-3.38)$ & 0.90 \\
\hline T4 & $1.33(0.29-5.98)$ & 0.707 & $1.19(0.37-3.73)$ & 0.76 \\
\hline TX & $0.67(0.11-4.00)$ & 0.666 & $1.36(0.39-4.70)$ & 0.63 \\
\hline \multicolumn{5}{|l|}{ N Staging } \\
\hline NO & 1.00 & & 1.00 & \\
\hline N1 & $1.07(0.70-1.63)$ & 0.743 & $1.54(1.34-1.77)$ & $<0.001$ \\
\hline $\mathrm{N} 2$ & $1.08(0.75-1.55)$ & 0.651 & $1.72(1.52-1.94)$ & $<0.001$ \\
\hline N3 & $0.88(0.26-2.93)$ & 0.836 & $2.12(1.49-3.03)$ & $<0.001$ \\
\hline NX & $1.30(0.17-9.88)$ & 0.795 & $1.19(0.75-1.89)$ & 0.45 \\
\hline \multicolumn{5}{|l|}{ Insurance Status } \\
\hline Not Insured & 1.00 & & 1.00 & \\
\hline Private Insurance/Managed Care & $0.74(0.42-1.32)$ & 0.319 & $0.81(0.65-1.00)$ & 0.06 \\
\hline Medicaid & $1.82(0.96-3.43)$ & 0.064 & $1.27(1.00-1.60)$ & 0.043 \\
\hline Medicare & $1.32(0.72-2.41)$ & 0.355 & $1.04(0.83-1.30)$ & 0.72 \\
\hline Other Government & $0.83(0.31-2.19)$ & 0.713 & $1.17(0.78-1.76)$ & 0.44 \\
\hline \multicolumn{5}{|l|}{ Median Income Quartiles 2008-2012 } \\
\hline$<\$ 38,000$ & 1.00 & & 1.00 & \\
\hline$\$ 38,000-\$ 47,999$ & $1.38(0.88-2.17)$ & 0.160 & $0.84(0.73-0.96)$ & 0.02 \\
\hline$\$ 48,000-\$ 62,999$ & $1.49(0.96-2.33)$ & 0.075 & $0.90(0.79-1.03)$ & 0.14 \\
\hline$\$ 63,000+$ & $1.37(0.87-2.16)$ & 0.169 & $0.77(0.67-0.88)$ & $<0.001$ \\
\hline \multicolumn{5}{|l|}{ Treatment Group } \\
\hline No treatment & 1.00 & & 1.00 & \\
\hline Radiation only & $0.56(0.25-1.23)$ & 0.151 & $0.49(0.37-0.63)$ & $<0.001$ \\
\hline Radiation and Chemo & $0.42(0.22-0.82)$ & 0.011 & $0.44(0.35-0.55)$ & $<0.001$ \\
\hline Surgery and Radiation & $0.23(0.10-0.50)$ & $<0.001$ & $0.33(0.26-0.43)$ & $<0.001$ \\
\hline Surgery, Chemotherapy and Radiation & $0.59(0.30-1.15)$ & 0.127 & $0.42(0.33-0.53)$ & $<0.001$ \\
\hline Surgery only & $0.48(0.24-0.94)$ & 0.033 & $0.37(0.29-0.45)$ & $<0.001$ \\
\hline Chemotherapy Only & $1.18(0.49-2.82)$ & 0.710 & $0.84(0.61-1.16)$ & 0.31 \\
\hline
\end{tabular}

\footnotetext{
${ }^{a}$ Insufficient sample size
} 
Interestingly, in our OCSCC study population, women were shown to have better survival than men in both the HPV-associated and HPV- group. This finding contrasts with the role that sex plays in OPSCCs and is consistent with the developing hypothesis that $\mathrm{OP}$ and non-OP SCCs are distinct cancers. Risk factors for OCSCC are well established: alcohol, tobacco and betel nut chewing $[2,24]$. Current rates of tobacco usage in the US are lower in women than in men [25]. As a result, a lower overall lifetime exposure to tobacco may partly explain the survival advantage among women in OCSCC. There is a new growing body of research interested in characterizing HPV in non-OP sites. A molecular study of 520 HNSCCs profiling the gene-expression signature of $\mathrm{HPV}$-associated OP and non-OP sites found there to be two distinct tumor immune microenvironments [8].

While our study did not directly test for the role of HPV within the OCSCC group, the similarity in risk factors between the HPV-associated and HPV- OCSCC groups infers that HPV may only play a minor prognostic role in OC cancers. A recent study by our group [26] found HPV to be associated with improved survival at the OCSCC subsite, though the survival advantage noted at the oral cavity subsite was not as great as that at the oropharynx subsite.

In our study, we found women were generally diagnosed with earlier $\mathrm{T}$ and $\mathrm{N}$ staged cancers than men. Earlier detection of cancers would lead to better prognosis [27]. From a health behavior perspective, this finding may be explained by the consistent underutilization of preventative healthcare by men leading to a delay in early diagnosis $[28,29]$. It has been hypothesized that women have more frequent contact with healthcare professionals due to pregnancy, childcare and hormone replacement therapy as well as women having more interest in health $[28,30]$.

The NCDB database, as a source, has well-documented limitations [31]. We were unable to account for every variable that may influence survival (e.g. alcohol, tobacco use, and other comorbidities), as these data were not captured by NCDB. In addition, the database does not capture other causes of OC and OP cancers that may influence survival. Specifically, studies have shown that patients with cancer from previous leukoplakia [32] or oral mucositis [33] leading to earlier cancer detection is associated with improved survival, where as patients with cancer from immunosuppression [34] tend to have worse survival. The type of testing (PCR, ISH for HPV DNA vs. p16) for HPV status may vary depending on each institution and reporting agency. Furthermore, the source of the sample may not necessarily derive from the primary site. There are likely low rates of misclassification due to the nature of the registry of the data; however, any misclassification is likely to have been evenly distributed across our four subgroups. Our retrospective study focuses on OS, not cancer-specific survival. The absence of cause-specific survival data in NCDB makes in plausible that other causes of death such as treatment derived toxicities, secondary primary cancer and comorbid cardiovascular, pulmonary and metabolic syndrome causes which are more prominent in men may contribute to the difference in mortality seen between the two sexes. In addition, other general cancer risk factors such as tobacco and alcohol as well as high-risk sex behavior associated with HPV+ transmission [35] may also influence the survival difference seen.

\section{Conclusion}

In summary, the effect of sex on outcomes of OP and OC SCCs appears to vary based on primary tumor location and HPV status. Notably, sex does not appear to affect the prognosis of HPV-associated OPSCCs after accounting for other risk factors. Men with HPV- OPSCCs appear to have a better prognosis for survival than women, though women appear to have a better prognosis in OCSCCs regardless of HPV-status. Given these results, we recommend further studies to investigate the clinical behavior and the sex-specific pathophysiological biology of HPV-associated HNSCCs and explore opportunities to further eliminate disparities in our patients.

\section{Abbreviations \\ HNSCC: Head and neck squamous cell carcinomas; HPV: Human papilloma virus; NCDB: National Cancer Database; OC: Oral cavity; OP: Oropharynx;} OS: Overall survival; SCC: Squamous cell carcinomas

\section{Funding}

William U. Gardner Memorial Student Research Fellowship at Yale University School of Medicine.

\section{Availability of data and materials}

The data that support the findings of this study are available from the Commission on Cancer's National Cancer Database. Restrictions apply to the availability of these data, which were used under license for the current study, and so are not publicly available. Access to the National Cancer Database can be found here: https://www.facs.org/quality-programs/cancer/ncdb.

\section{Authors' contributions}

HL: Conceptualization, methodology, software, validation, formal analysis, investigation, resources, data curation, writing-original draft, and visualization. HSP: Writing-review and editing, visualization. HAO: Writingreview and editing. BLJ: Conceptualization, methodology, formal analysis, investigation, writing-review and editing, supervision, project administration, and funding acquisition. All authors read and approved the final manuscript.

\section{Ethics approval and consent to participate}

Our study is exempt from review by the Yale Human Research Protection

Program because it uses a pre-existing, de-identified public database.

\section{Consent for publication}

Not applicable.

Competing interests

The authors declare that they have no competing interests.

\section{Publisher's Note}

Springer Nature remains neutral with regard to jurisdictional claims in published maps and institutional affiliations. 


\section{Author details}

${ }^{1}$ Yale School of Medicine, New Haven, CT, USA. ${ }^{2}$ Department of Therapeutic Radiology, Yale New Haven Hospital, New Haven, CT, USA. ${ }^{3}$ Department of Surgery (Section of Otolaryngology), Yale New Haven Hospital, New Haven, CT, USA. ${ }^{4}$ Yale Cancer Center, 330 Cedar Street, PO Box 208062, New Haven, CT 06520-8062, USA

Received: 8 February 2018 Accepted: 29 May 2018

Published online: 20 June 2018

\section{References}

1. Gillison ML. Human papillomavirus-associated head and neck cancer is a distinct epidemiologic, clinical, and molecular entity. Semin Oncol. 2004;31: 744-54. https://doi.org/10.1053/j.seminoncol.2004.09.011.

2. Blot WJ, McLaughlin JK, Winn DM, Austin DF, Greenberg RS, Preston-Martin $\mathrm{S}$, et al. Smoking and drinking in relation to oral and pharyngeal Cancer. Cancer Res. 1988;48:3282. http://cancerres.aacrjournals.org/content/48/11/ 3282.abstract

3. Chaturvedi AK, Engels EA, Anderson WF, Gillison ML. Incidence trends for human papillomavirus-related and -unrelated oral squamous cell carcinomas in the United States. J Clin Oncol Off J Am Soc Clin Oncol. 2008:26:612-9.

4. D'Souza G, Westra WH, Wang SJ, van Zante A, Wentz A, Kluz N, et al. Differences in the prevalence of human papillomavirus (HPV) in head and neck squamous cell cancers by sex, race, anatomic tumor site, and HPV detection method. JAMA Oncol. 2017;3:169. https://doi.org/10.1001/ jamaoncol.2016.3067.

5. Chung $\mathrm{CH}$, Zhang Q, Kong CS, Harris J, Fertig EJ, Harari PM, et al. p16 protein expression and human papillomavirus status as prognostic biomarkers of nonoropharyngeal head and neck squamous cell carcinoma. J Clin Oncol. 2014;32:3930-8. https://doi.org/10.1200/JCO.2013.54.5228.

6. Herrero R, Castellsagué X, Pawlita M, Lissowska J, Kee F, Balaram P, et al. Human papillomavirus and oral cancer: the International Agency for Research on Cancer multicenter study. J Natl Cancer Inst. 2003;95:1772-83.

7. Lassen P, Primdahl H, Johansen J, Kristensen CA, Andersen E, Andersen LJ, et al. Impact of HPV-associated p16-expression on radiotherapy outcome in advanced oropharynx and non-oropharynx cancer. Radiother Oncol J Eur Soc Ther Radiol Oncol. 2014;113:310-6.

8. Chakravarthy A, Henderson S, Thirdborough SM, Ottensmeier $\mathrm{CH}$, Su X, Lechner $M$, et al. Human papillomavirus drives tumor development throughout the head and neck: improved prognosis is associated with an immune response largely restricted to the oropharynx. J Clin Oncol. 2016; 34:4132-41. https://doi.org/10.1200/JCO.2016.68.2955.

9. Fakhry C, Westra WH, Wang SJ, van Zante A, Zhang Y, Rettig E, et al. The prognostic role of sex, race, and human papillomavirus in oropharyngeal and nonoropharyngeal head and neck squamous cell cancer: role of sex, race, and HPV in HNSCC prognosis. Cancer. 2017;123:1566-75. https://doi. org/10.1002/cncr.30353.

10. Fakhry C, Westra WH, Li S, Cmelak A, Ridge JA, Pinto H, et al. Improved survival of patients with human papillomavirus-positive head and neck squamous cell carcinoma in a prospective clinical trial. J Natl Cancer Inst. 2008;100:261-9.

11. Gillison ML, Chaturvedi AK, Anderson WF, Fakhry C. Epidemiology of human papillomavirus-positive head and neck squamous cell carcinoma. J Clin Oncol Off J Am Soc Clin Oncol. 2015;33:3235-42.

12. About the National Cancer Database. American College of Surgeons. https:// www.facs.org/quality-programs/cancer/ncdb/about. Accessed 20 Sep 2017.

13. Goon PKC, Stanley MA, Ebmeyer J, Steinsträsser L, Upile T, Jerjes W, et al. HPV \& head and neck cancer: a descriptive update. Head Neck Oncol. 2009;1:36.

14. Ang KK, Harris J, Wheeler R, Weber R, Rosenthal DI, Nguyen-Tân PF, et al. Human papillomavirus and survival of patients with oropharyngeal cancer. N Engl J Med. 2010;363:24-35.

15. Rintala M, Grénman S, Puranen M, Syrjänen S. Natural history of oral papillomavirus infections in spouses: a prospective Finnish HPV family study. $\mathrm{J}$ Clin Virol Off Publ Pan Am Soc Clin Virol. 2006;35:89-94.

16. Lorenzi A, Rautava J, Kero K, Syrjänen K, Longatto-Filho A, Grenman S, et al. Physical state and copy numbers of HPV16 in oral asymptomatic infections that persisted or cleared during the 6-year follow-up. J Gen Virol. 2017;98: 681-9. https://doi.org/10.1099/jgv.0.000710.
17. Tsakogiannis D, Kyriakopoulou Z, Ruether IGA, Amoutzias GD, Dimitriou TG, Diamantidou $V$, et al. Determination of human papillomavirus 16 physical status through E1/E6 and E2/E6 ratio analysis. J Med Microbiol. 2014;63(Pt 12):1716-23.

18. Briolat J, Dalstein V, Saunier M, Joseph K, Caudroy S, Prétet J-L, et al. HPV prevalence, viral load and physical state of HPV-16 in cervical smears of patients with different grades of CIN. Int J Cancer. 2007;121:2198-204.

19. Cook MB, McGlynn KA, Devesa SS, Freedman ND, Anderson WF. Sex disparities in cancer mortality and survival. Cancer Epidemiol Biomark Prev Publ Am Assoc Cancer Res Cosponsored Am Soc Prev Oncol. 2011;20:1629-37.

20. Scosyrev E, Noyes K, Feng C, Messing E. Sex and racial differences in bladder cancer presentation and mortality in the US. Cancer. 2009;115:68-74.

21. Shariat SF, Sfakianos JP, Droller MJ, Karakiewicz PI, Meryn S, Bochner BH. The effect of age and gender on bladder cancer: a critical review of the literature. BJU Int. 2010;105:300-8.

22. Yuan F, Auborn K, James C. Altered growth and viral gene expression in human papillomavirus type 16-containing cancer cell lines treated with progesterone. Cancer Investig. 1999;17:19-29.

23. Newfield L, Goldsmith A, Bradlow HL, Auborn K. Estrogen metabolism and human papillomavirus-induced tumors of the larynx: chemo-prophylaxis with indole-3-carbinol. Anticancer Res. 1993;13:337-41.

24. Warnakulasuriya S, Trivedy C, Peters TJ. Areca nut use: an independent risk factor for oral cancer. BMJ. 2002;324:799-800. https://www.ncbi.nlm.nih.gov/ pmc/articles/PMC1122751/. Accessed 18 Oct 2017

25. Health CO on S and. Smoking and Tobacco Use; Fact Sheet; Adult Cigarette Smoking in the United States; Smoking and Tobacco Use. http://www.cdc. gov/tobacco/data_statistics/fact_sheets/adult_data/cig_smoking/. Accessed 18 Oct 2017.

26. Li H, Torabi S, Yarbrough WG, Mehra S, Osborn HA, Judson BL. Association of Human Papillomavirus Status with Overall Survival at head and neck carcinoma subsites. JAMA Otolaryngol Neck Surg. 144:1-7.

27. Cancer Screening Overview. National Cancer Institute https://www. cancer.gov/about-cancer/screening/hp-screening-overview-pdq. Accessed 22 Apr 2018.

28. Green CA, Pope CR. Gender, psychosocial factors and the use of medical services: a longitudinal analysis. Soc Sci Med. 1999;48:1363-72.

29. Bertakis KD, Azari R, Helms LJ, Callahan EJ, Robbins JA. Gender differences in the utilization of health care services. J Fam Pract. 2000;49:147-52.

30. Evans R, Brotherstone H, Miles A, Wardle J. Gender differences in early detection of cancer. J Men's Health Gend. 2:209-17.

31. Boffa DJ, Rosen JE, Mallin K, Loomis A, Gay G, Palis B, et al. Using the National Cancer Database for outcomes research: a review. JAMA Oncol. 2017; https://doi.org/10.1001/jamaoncol.2016.6905.

32. Yanik EL, Katki HA, Silverberg MJ, Manos MM, Engels EA, Chaturvedi AK. Leukoplakia, oral cavity Cancer risk, and Cancer survival in the U.S. elderly. Cancer Prev Res Phila Pa. 2015;8:857-63.

33. Rastogi M, Dwivedi RC, Kazi R. Oral mucositis in head and neck cancer. Eur J Cancer Care (Engl). 2011;20:144.

34. Deeb R, Sharma S, Mahan M, Al-Khudari S, Hall F, Yoshida A, et al. Head and neck cancer in transplant recipients. Laryngoscope. 2012;122:1566-9.

35. Giuliano AR, Tortolero-Luna G, Ferrer E, Burchell AN, de Sanjose S, Kjaer SK, et al. Epidemiology of human papillomavirus infection in men, cancers other than cervical and benign conditions. Vaccine. 2008;26:K17-28. https:// doi.org/10.1016/j.vaccine.2008.06.021.

\section{Ready to submit your research? Choose BMC and benefit from:}

- fast, convenient online submission

- thorough peer review by experienced researchers in your field

- rapid publication on acceptance

- support for research data, including large and complex data types

- gold Open Access which fosters wider collaboration and increased citations

- maximum visibility for your research: over $100 \mathrm{M}$ website views per year

At BMC, research is always in progress.

Learn more biomedcentral.com/submissions 